\title{
Gentisate and 3-oxoadipate pathways in the yeast Candida parapsilosis: identification and functional analysis of the genes coding for 3-hydroxybenzoate 6-hydroxylase and 4-hydroxybenzoate 1-hydroxylase \\ Correspondence \\ Jozef Nosek \\ nosek@fns.uniba.sk \\ Received 12 January 2011 \\ Revised 29 March 2011 \\ Accepted 5 April 2011

\author{
Zuzana Holesova, ${ }^{1,2}$ Michaela Jakubkova, ${ }^{1}$ Ivana Zavadiakova, ${ }^{2}$ \\ Igor Zeman, ${ }^{1}$ Lubomir Tomaska ${ }^{2}$ and Jozef Nosek $^{1}$
}

\author{
${ }^{1}$ Department of Biochemistry, Comenius University in Bratislava, Faculty of Natural Sciences, \\ Mlynska dolina $\mathrm{CH}-1,84215$ Bratislava, Slovak Republic \\ dolina B-1, 84215 Bratislava, Slovak Republic
} \\ ${ }^{2}$ Department of Genetics, Comenius University in Bratislava, Faculty of Natural Sciences, Mlynska

\begin{abstract}
The pathogenic yeast Candida parapsilosis degrades various hydroxy derivatives of benzenes and benzoates by the gentisate and 3-oxoadipate pathways. We identified the genes MNX1, MNX2, $M N X 3, G D X 1, H D X 1$ and $F P H 1$ that code for enzymes involved in these pathways in the complete genome sequence of $C$. parapsilosis. Next, we demonstrated that MNX1, MNX2, MNX3 and $G D X 1$ are inducible and transcriptionally controlled by hydroxyaromatic substrates present in cultivation media. Our results indicate that MNX1 and MNX2 code for flavoprotein monooxygenases catalysing the first steps in the 3-oxoadipate and gentisate pathways, respectively (i.e. 4-hydroxybenzoate 1-hydroxylase and 3-hydroxybenzoate 6-hydroxylase). Moreover, we found that the two pathways differ by their intracellular localization. The enzymes of intracellular localization of the components of the gentisate pathway, Mnx2p and Gdx1p, depends on the substrate in the cultivation medium. In cells growing on glucose these proteins localize in the cytosol, whereas in media containing hydroxyaromatic compounds they associate with mitochondria. Finally, we showed that the overexpression of MNX1 or MNX2 increases the tolerance of C. parapsilosis cells to the antifungal drug terbinafine.
\end{abstract} the 3-oxoadipate pathway, Mnx1p and Mnx3p, localize predominantly in the cytosol. In contrast,
}

\section{INTRODUCTION}

Candida parapsilosis is a pathogenic yeast with increasing medical importance, which exhibits a number of peculiar features (Nosek et al., 2009; Trofa et al., 2008; van Asbeck et al., 2009; Weems, 1992). This species is commonly isolated from environmental sources such as soil, plants and plant-associated insects (Carruba et al., 1991; Suh et al., 2008; Trindade et al., 2002), suggesting that it participates in decomposition of decaying plant tissues including hemicellulose and lignin degradation products. This is in line with its ability to ferment D-xylose and to utilize diverse phenol derivatives as the sole carbon sources. Understanding the metabolism of phenolic compounds may be important for biotechnological applications and/or bioremediation. In C. parapsilosis, hydroxybenzenes and hydroxybenzoates are degraded via a hydroxyhydroquinone (HHQ, 1,2,4-trihydroxybenzene) variant of the 3-

Abbreviations: GSH, glutathione; $\mathrm{HHO}$, hydroxyhydroquinone.

Two supplementary figures and a supplementary table are available with the online version of this paper. oxoadipate pathway and a glutathione (GSH)-dependent variant of the gentisate pathway (Fig. 1). In the first pathway, 4-hydroxybenzoate 1-hydroxylase (Mnx1p) decarboxylates 4-hydroxybenzoate to hydroquinone (1,4dihydroxybenzene), which is a substrate for hydroquinone hydroxylase (phenol 2-monooxygenase, Mnx3p). Both enzymes are $\mathrm{NAD}(\mathrm{P}) \mathrm{H}$-dependent flavoprotein monooxygenases with broad substrate specificity. 4-Hydroxybenzoate 1-hydroxylase utilizes 4-hydroxybenzoate, resorcinate (2,4dihydroxybenzoate) and protocatechuate (3,4-dihydroxybenzoate); and hydroquinone hydroxylase uses hydroquinone and resorcinol (1,3-dihydroxybenzene). These substrates are converted to HHQ, which is further metabolized via 2-maleylacetate and 3-oxoadipate to succinate and acetylCoA. In the second pathway, 3-hydroxybenzoate is hydroxylated by 3-hydroxybenzoate 6-monooxygenase (Mnx2p) to gentisate (2,5-dihydroxybenzoate), which is converted by gentisate 1,2-dioxygenase (Gdxlp) to 3-maleylpyruvate and subsequently metabolized via 3-fumarylpyruvate to fumarate and pyruvate (Cooper \& Land, 1979; Middelhoven, 1993; Middelhoven et al., 1992; van Berkel et al., 1994). 


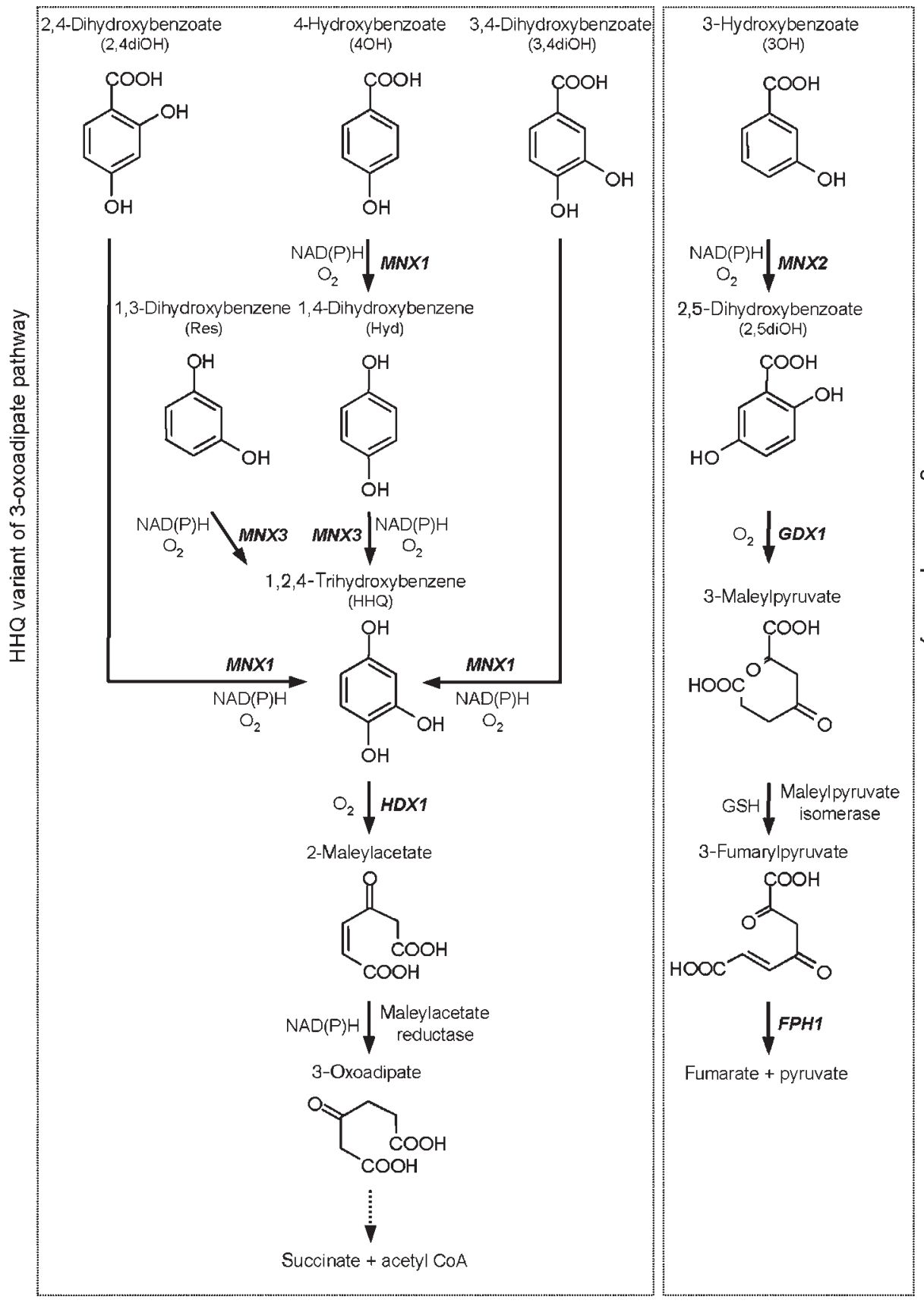

Fig. 1. Catabolic pathways involved in the degradation of hydroxybenzoates and hydroxybenzenes operating in C. parapsilosis cells. Identified genes coding for enzymes catalysing the corresponding reactions are indicated in bold; MNX1 (CPAG01781), MNX2 (CPAG03410), MNX3 (CPAG00562), GDX1 (CPAG03408), HDX1 (CPAG05217), FPH1 (CPAG03407). Note that Eppink et al. $(1997,2000)$ characterized the enzymatic activities of Mnx1p and Mnx3p. Abbreviations of the phenolic compounds are shown in parentheses.

In this study, we addressed two questions: (i) what is the genetic basis of utilization of hydroxybenzenes and hydroxybenzoates in C. parapsilosis; and (ii) what is the occurrence of the corresponding genes in related yeast species. We took advantage of the complete genome sequences of $C$. parapsilosis and closely related species from the 'CTG' clade of hemiascomycetes (Butler et al., 2009; Jeffries et al., 2007; Jones et al., 2004) and searched for candidate genes coding for the enzymes involved in both metabolic pathways mentioned above. We identified open reading frames (ORFs) coding for components of the 3-oxoadipate and gentisate pathways and we named 
them MNX1 (monooxygenase 1; systematic ORF name in the C. parapsilosis genome database CPAG01781), MNX2 (monooxygenase 2; CPAG03410), MNX3 (monooxygenase 3; CPAG00562), GDX1 (gentisate dioxygenase 1; CPAG03408), HDX1 (hydroxyquinol dioxygenase 1; CPAG05217) and FPH1 (fumarylpyruvate hydrolase 1; CPAG03407). Next, we demonstrated that MNX1, MNX2 and MNX3 code for flavoprotein monooxygenases with the activity of 4-hydroxybenzoate 1-hydroxylase, 3-hydroxybenzoate 6hydroxylase and hydroquinone hydroxylase, respectively. Comparative analysis revealed that most of the examined yeast species lack homologues of one or more genes present in C. parapsilosis, which is underlined by their inability to grow in media containing the corresponding hydroxybenzenes or hydroxybenzoates as the sole carbon source.

\section{METHODS}

Yeast strains and cultivation. C. parapsilosis strains CLIB214 (the wild-type strain; identical to CBS604 ${ }^{\mathrm{T}}$ ), CDU1 (CLIB214 ura3 $:$ : FRT/ura3 $\Delta:$ :FRT; Ding \& Butler, 2007), SR23 (=CBS7157; ade ${ }^{-} y_{s} 4^{-}$) and SR23 met-1 (ade lys $4^{-}$met $2^{-}$; Nosek et al., 2002) were used for experiments described in this study. C. albicans CBS562 ${ }^{\mathrm{NT}}, C$. dubliniensis CBS7987 ${ }^{\mathrm{T}}$, C. glabrata CBS138 ${ }^{\mathrm{T}}$, C. metapsilosis $\mathrm{CBS}_{10907^{\mathrm{T}}}$ $\left(\mathrm{MCO} 448^{\mathrm{T}}\right)$, C. orthopsilosis CBS10906 ${ }^{\mathrm{T}}\left(\mathrm{MCO} 457^{\mathrm{T}}\right)$, C. parapsilosis $\mathrm{CBS}_{604}{ }^{\mathrm{T}}$, C. tropicalis CBS94 ${ }^{\mathrm{T}}$, Clavispora lusitaniae $\mathrm{CBS}_{6936^{\mathrm{T}}}$, Debaryomyces hansenii $\mathrm{CBS}_{76} 7^{\mathrm{T}}$, Lodderomyces elongisporus $\mathrm{CBS} 2605^{\mathrm{T}}$, Pichia guilliermondii CBS2030 ${ }^{\mathrm{T}}$, Pichia stipitis $\mathrm{CBS}_{5773^{\mathrm{T}}}$ and Saccharomyces cerevisiae L5366 (MATa/ $\alpha$ ura3-52/ura3-52; Gimeno \& Fink, 1994) were used in assimilation tests of hydroxyaromatic compounds. Yeasts were cultivated in media listed in Table 1.

Plasmid constructs. The coding sequences of $C$. parapsilosis genes MNX1, MNX2, MNX3 and GDX1 were amplified with or without the termination codon from the genomic DNA of strain CBS604 by using
PCR with the primers listed in Table 2. The PCR products were cloned into pGEM-T Easy (Promega) or pDrive (Qiagen) vectors. The resulting plasmid constructs were then digested with the endonuclease $X b a \mathrm{I}$ and the DNA fragments coding for investigated proteins were inserted into the XbaI site of pBP7 and pBP8 (Kosa et al., 2007). The gene promoters were amplified as PCR products derived 747$1051 \mathrm{bp}$ upstream of the corresponding ORF, phosphorylated with T4 polynucleotide kinase and cloned into the pPK3 vector (Kosa et al., 2007) linearized with the endonuclease SmaI. The inserts in all plasmid constructs were verified by DNA sequencing and comparison with the genome sequence of the $C$. parapsilosis reference strain CDC317 (http://www.sanger.ac.uk/sequencing/Candida/parapsilosis/). All plasmid constructs are shown in Table 3. The plasmid DNA was transformed into C. parapsilosis cells by lithium acetate/polyethylene glycol/carrier DNA protocol (Nosek et al., 2002) or by electroporation (Zemanova et al., 2004).

NADPH oxidation assays. Yeast cells grown in synthetic media (Table 1) were harvested at late exponential phase by centrifugation $\left(10 \mathrm{~min}, 2500 \mathrm{~g}\right.$ at $4{ }^{\circ} \mathrm{C}$ ), washed with water and resuspended in 5 vols $50 \mathrm{mM}$ Tris/ $\mathrm{HCl}, 5 \mathrm{mM}$ EDTA ( $\mathrm{pH}$ 8.0). Samples were vortexed with glass beads $\left(0.45-0.5 \mathrm{~mm}\right.$ in diameter, $\left.0.8 \mathrm{~g} \mathrm{ml}^{-1}\right)$ four times for $1 \mathrm{~min}$ with $1 \mathrm{~min}$ intermissions on ice. After centrifugation $(10 \mathrm{~min}$, $1500 \mathrm{~g}$ at $4{ }^{\circ} \mathrm{C}$ ) the supernatant was transferred to a new Eppendorf tube. Enzymatic activities of Mnx1p, Mnx2p and Mnx3p in cell lysates were measured essentially as described by Eppink et al. (1997). Briefly, 250-500 $\mu \mathrm{g}$ protein extract was added to $50 \mathrm{mM}$ potassium phosphate buffer ( $\mathrm{pH}$ 7.6) containing $0.2 \mathrm{mM}$ NADPH, $10 \mu \mathrm{M}$ FAD and $1 \mathrm{mM}$ of appropriate substrate. Oxidation of NADPH at $340 \mathrm{~nm}$ and $25{ }^{\circ} \mathrm{C}$ was continuously monitored by using a Hitachi U-2001 spectrophotometer. The amount of oxidized NADPH $\mathrm{min}^{-1}$ (mg protein $)^{-1}$ was calculated from the decrease in the absorbance at defined time intervals.

Oxygen consumption measurements. Yeast cells grown in synthetic media (Table 1) were harvested at late exponential phase by centrifugation $\left(10 \mathrm{~min}, 2500 \mathrm{~g}\right.$ at $4{ }^{\circ} \mathrm{C}$ ), washed twice with water, then with $40 \mathrm{mM}$ potassium phosphate buffer $(\mathrm{pH} \mathrm{7.4)}$ and

Table 1. Yeast cultivation media

Agar (2\%) was added for solid media. YNB-AA-AS, yeast nitrogen base without amino acids and ammonium sulphate (Difco). Synthetic media (S) were supplemented with $20 \mu \mathrm{g}$ adenine $\mathrm{ml}^{-1}$ and $50 \mu \mathrm{g}$ lysine ml $\mathrm{ml}^{-1}$ for cultivation of strains SR23 and SR23 met-1. Hydroxyaromatic compounds were dissolved in DMSO as 0.5 or $1 \mathrm{M}$ stocks. All percentage values are w/v.

\begin{tabular}{|l|c|}
\hline Name & Composition \\
\hline YPD & $1 \%$ yeast extract, $2 \%$ peptone, $2 \%$ glucose \\
SD & $0.17 \%$ YNB-AA-AS, $0.5 \%\left(\mathrm{NH}_{4}\right)_{2} \mathrm{SO}_{4}, 2 \%$ glucose \\
SGal & $0.17 \%$ YNB-AA-AS, $0.5 \%\left(\mathrm{NH}_{4}\right)_{2} \mathrm{SO}_{4}, 2 \%$ galactose \\
SGly & $0.17 \%$ YNB-AA-AS, $0.5 \%\left(\mathrm{NH}_{4}\right)_{2} \mathrm{SO}_{4}, 3 \%$ glycerol \\
S2OH & $0.17 \%$ YNB-AA-AS, $0.5 \%\left(\mathrm{NH}_{4}\right)_{2} \mathrm{SO}_{4}, 10 \mathrm{mM}$ salicylate \\
SAsp & $0.17 \%$ YNB-AA-AS, $0.5 \%\left(\mathrm{NH}_{4}\right)_{2} \mathrm{SO}_{4}, 10 \mathrm{mM}$ aspirin \\
S3OH & $0.17 \%$ YNB-AA-AS, $0.5 \%\left(\mathrm{NH}_{4}\right)_{2} \mathrm{SO}_{4}, 10 \mathrm{mM}$-hydroxybenzoate \\
S4OH & $0.17 \%$ YNB-AA-AS, $0.5 \%\left(\mathrm{NH}_{4}\right)_{2} \mathrm{SO}_{4}, 10 \mathrm{mM}$-hydroxybenzoate \\
S2,4diOH & $0.17 \%$ YNB-AA-AS, $0.5 \%\left(\mathrm{NH}_{4}\right)_{2} \mathrm{SO}_{4}, 10 \mathrm{mM}$ resorcinate \\
S3,4diOH & $0.17 \%$ YNB-AA-AS, $0.5 \%\left(\mathrm{NH}_{4}\right)_{2} \mathrm{SO}_{4}, 10 \mathrm{mM}$ protocatechuate \\
S2,5diOH & $0.17 \%$ YNB-AA-AS, $0.5 \%\left(\mathrm{NH}_{4}\right)_{2} \mathrm{SO}_{4}, 10 \mathrm{mM}$ gentisate \\
SCat & $0.17 \%$ YNB-AA-AS, $0.5 \%\left(\mathrm{NH}_{4}\right)_{2} \mathrm{SO}_{4}, 10 \mathrm{mM}$ catechol \\
SRes & $0.17 \%$ YNB-AA-AS, $0.5 \%\left(\mathrm{NH}_{4}\right)_{2} \mathrm{SO}_{4}, 10 \mathrm{mM}$ resorcinol \\
SHyd & $0.17 \%$ YNB-AA-AS, $0.5 \%\left(\mathrm{NH}_{4}\right)_{2} \mathrm{SO}_{4}, 10 \mathrm{mM}$ hydroquinone \\
\hline
\end{tabular}


Table 2. Oligonucleotide primers

\begin{tabular}{|ll|}
\hline Name & \multicolumn{1}{c|}{ Sequence $\left(\mathbf{5}^{\prime} \rightarrow \mathbf{3}^{\prime}\right)$} \\
\hline Mnx1up_XbaI & TCTAGAATGGCAGTTCAAGCACCAT \\
Mnxidn_XbaI & TCTAGATTAGCCTGATGCACTTAATGGA \\
Mnx1dn_XbaI_w/o_Stop & TCTAGAGCCTGATGCACTTAATGGA \\
MnxlupProm & TTGTTGGTATAAGCAAAGAGC \\
MnxidnProm & CATTTCTCTGGTGTGTACTTGT \\
Mnx2up_XbaI & TCTAGAATGAATCTGCTCTCATTT \\
Mnx2dn_XbaI & TCTAGACTATAACTTAGATTCAAAAGT \\
Mnx2dn_XbaI_w/o_Stop & TCTAGATAACTTAGATTCAAAAGTACTG \\
Mnx2upProm & TCATTGTGTTCTTGTGGTAA \\
Mnx2dnProm & CATGAACTTATATATATAGTTTCATG \\
Mnx3up_XbaI & TCTAGAATGCCCTCACAACAAACA \\
Mnx3dn_XbaI & TCTAGATTATACTGCTAATCTTGGT \\
Mnx3dn_XbaI_w/o_Stop & TCTAGATACTGCTAATCTTGGTTGAAG \\
Mnx3upProm & TTATGGTTTGTAGCCCTGTTG \\
Mnx3dnProm & CATGTTGTTTGTGAAGTTTGGT \\
Gdxlup_XbaI & TCTAGAATGTCTCCAGTCGAAGCATC \\
Gdx1dn_XbaI & TCTAGATTAATTTGCAGCAGTTTTGTG \\
Gdx1dn_XbaI_w/o_Stop & TCTAGAATTTGCAGCAGTTTTGTGGAT \\
GdxlupProm & CGTAGCTTAAAGCATCTTCTTC \\
Gdx1dnProm & CATTCTAAATGATTCTTGAGTGA \\
& \\
\hline
\end{tabular}

resuspended in the same buffer. Oxygen consumption was measured at $30{ }^{\circ} \mathrm{C}$ in a thermostatically controlled chamber equipped with a Clark electrode. Oxygen depletion by endogenous respiration and after addition of $10 \mathrm{mM}$ phenolic substrates was recorded and analysed by using an Oxygen meter 782 with data analysis module (Strathkelvin Instruments).

Fluorescence microscopy. Intracellular localization of the proteins Mnx1, Mnx2, Mnx3 and Gdx1 was observed in C. parapsilosis CDU1 cells transformed with pBP8-MNX1, pBP8-MNX2, pBP8-MNX3 and pBP8-GDX1 plasmids expressing the proteins fused with the enhanced version 3 of green fluorescent protein (yEGFP3). To visualize the fusion proteins, the transformants were cultivated overnight at $28{ }^{\circ} \mathrm{C}$ in synthetic media containing various carbon sources and observed by using a BX50 microscope equipped with the appropriate filter set and digital camera DP70 (Olympus). Mitochondria were counterstained with $25 \mathrm{nM}$ MitoTracker Red CMXRos (Molecular Probes) for 15-60 min.

Bioinformatic analyses. The gene sequences of $C$. parapsilosis, their systematic names and homologues were identified using the BLAST tools at the C. parapsilosis and C. dubliniensis sequencing sites (http:// www.sanger.ac.uk/sequencing/Candida/parapsilosis/; http://www. sanger.ac.uk/sequencing/Candida/dubliniensis/), Candida comparative site (http://www.broadinstitute.org/annotation/genome/candida_group/ MultiHome.html), P. stipitis v2.0 database (http://genome.jgi-psf.org/ Picst3/Picst3.home.html) and the National Center for Biotechnology Information (http://www.ncbi.nlm.nih.gov/). Syntenic context was examined using the Candida gene order browser (http://cgob.ucd.ie/; Fitzpatrick et al., 2010).

\section{Table 3. Plasmid constructs}

C. parapsilosis CDU1 was the host strain for all pBP7 and pBP8 constructs, and C. parapsilosis SR23 met-1 was the host cell for all pPK3 constructs.

\begin{tabular}{|ll|}
\hline Name & \multicolumn{1}{c|}{ Expressed gene } \\
\hline pBP7-MNX1 & C. parapsilosis MNX1 driven by the GAL1 promoter \\
pBP7-MNX2 & C. parapsilosis MNX2 driven by the GAL1 promoter \\
pBP7-MNX3 & C. parapsilosis MNX3 driven by the GAL1 promoter \\
pBP8-MNX1 & C. parapsilosis MNX1 fused with the yEGFP3 ORF \\
pBP8-MNX2 & C. parapsilosis MNX2 fused with the yEGFP3 ORF \\
pBP8-MNX3 & C. parapsilosis MNX3 fused with the yEGFP3 ORF \\
pBP8-GDX1 & C. parapsilosis GDX1 fused with the yEGFP3 ORF \\
pPK3-P $P_{M N X 1}$ & K. lactis LAC4 driven by the MNX1 promoter \\
pPK3-P & K. lactis LAC4 driven by the MNX2 promoter \\
pPK3-P & K. lactis LAC4 driven by the MNX3 promoter \\
pPK3-P & KDX1 lactis LAC4 driven by the GDX1 promoter \\
\hline
\end{tabular}




\section{RESULTS AND DISCUSSION}

\section{Utilization of hydroxybenzoates and hydroxybenzenes by $\boldsymbol{C}$. parapsilosis and related yeast species}

C. parapsilosis does not utilize phenol, catechol (1,2dihydroxybenzene), salicylate (2-hydroxybenzoate) and 2,3-dihydroxybenzoate. However, it grows on several hydroxy derivatives of benzene and benzoate such as 3hydroxybenzoate, 4-hydroxybenzoate, gentisate, hydroquinone, protocatechuate, resorcinate and resorcinol (Middelhoven, 1993; Middelhoven et al., 1992). Catabolism of these compounds proceeds either via a HHQ variant of the 3-oxoadipate pathway or a GSH variant of the gentisate pathway (Fig. 1). In contrast with the $C$. parapsilosis type strain CBS604 (=CLIB214), we found that two laboratory strains, SR23 (=CBS7157) and SR23 met-1, do not grow on hydroquinone (data not shown). These strains were mutageniszed and we assume that SR23, which is parental to SR23 met-1, may have also acquired a mutation in a component of the 3-oxoadipate pathway.

In order to identify whether both pathways are conserved in other yeasts, we examined the growth of selected species in synthetic media containing hydroxybenzenes or hydroxybenzoates as the sole carbon sources (Fig. 2a). Our results show that, in contrast with $C$. parapsilosis, the other yeasts examined either do not grow (i.e. C. glabrata, $C$. lusitaniae, D. hansenii, S. cerevisiae) or utilize only a limited number of tested substrates (i.e. C. albicans, C. dubliniensis, C. metapsilosis, C. orthopsilosis, C. tropicalis, L. elongisporus, $P$. guilliermondii, $P$. stipitis), suggesting that the two pathways are compromised in these species. The 3-oxoadipate pathway appears to be conserved in C. orthopsilosis and $C$. metapsilosis, which were originally classified as C. parapsilosis groups II and III (Tavanti et al., 2005), respectively. Both species utilize 4-hydroxybenzoate, resorcinate, protocatechuate, hydroquinone and resorcinol. Interestingly, these species do not grow on 3-hydroxybenzoate and gentisate, indicating that they lack a functional gentisate pathway. This pathway appears to be present only in $C$. parapsilosis and P. stipitis. Interestingly, none of the examined yeasts grew in media containing $10 \mathrm{mM}$ salicylate as the sole carbon source. Since acetylsalicylate (aspirin) induces apoptosis-like cell death in S. cerevisiae (Balzan et al., 2004) and exhibits antifungal properties in a number of yeast species (Leeuw et al., 2007), we analysed whether the absence of growth of $C$. parapsilosis on salicylate is due to its inhibitory effect on the growth or inability of this yeast to utilize salicylate as the sole carbon source (Fig. 2b). We found that C. parapsilosis, as well as two other pathogenic species C. albicans and C. glabrata, are unable to grow in media with salicylate or acetylsalicylate (aspirin) even in combination with glucose or glycerol, suggesting that both hydroxybenzoates are cytotoxic. In addition, we observed that the treatment with salicylate or aspirin also reduces the viability of $C$. parapsilosis in nongrowth conditions (data not shown). Moreover, we noticed that the morphology of $C$. parapsilosis colonies developed from either single cells (dubbed 'clonal colonies') or cell suspensions ('macrocolonies') is a characteristic, substratedependent feature (Fig. 2c). This indicates that catabolic degradation of the hydroxyaromatic compounds investigated is associated with global cellular responses, reflected by changes in morphogenesis and/or cell differentiation.

Next, we examined the activities of enzymes catalysing the first steps of the gentisate and 3-oxoadipate pathways. 3Hydroxybenzoate 6-hydroxylase, 4-hydroxybenzoate 1hydroxylase and hydroquinone hydroxylase were analysed in protein extracts from C. parapsilosis cells cultivated in media containing glucose or phenolic substrate using the NADPH oxidation assay (Fig. 3a). We detected low enzymatic activities in extracts prepared from cells grown in SD medium. However, the activities were $\sim 20$ - to $>100$ fold higher in extracts prepared from cells grown in media with hydroxybenzoates or hydroxybenzenes, indicating that the corresponding enzymes are induced on these substrates. In a complementary experiment, we analysed the enzymes mentioned above as well as gentisate dioxygenase, which does not use $\mathrm{NAD}(\mathrm{P}) \mathrm{H}$ as a cofactor. We inferred the enzymatic activities from oxygen consumption of $C$. parapsilosis cells grown in synthetic media differing by the carbon source (Fig. 3b). Using this assay, we detected a substrate-dependent increase in oxygen consumption in cells cultivated in media containing hydroxyaromatic compounds but not in cells grown on glucose or glycerol. For example, cells cultivated on 3-hydroxybenzoate exhibited increased oxygen consumption after addition of 3-hydroxybenzoate (6.6-fold), gentisate (3.6-fold) and protocatechuate (2.6-fold). Similar enzymatic activities were found in cells utilizing gentisate as the carbon source. In contrast, cells grown on 4-hydroxybenzoate (and similarly on hydroquinone) had increased activities with 4-hydroxybenzoate (4.3fold), catechol (2-fold), resorcinol (3.2-fold), hydroquinone (4.5-fold), resorcinate (1.4-fold) and protocatechuate (4.3fold). These results indicate that the two pathways are regulated independently and appear to be induced by the corresponding substrate (i.e. the gentisate pathway by 3hydroxybenzoate and gentisate, and the 3-oxoadipate pathway by 4-hydroxybenzoate and hydroquinone).

\section{C. parapsilosis genes encoding the enzymes of the gentisate and 3-oxoadipate pathways}

To identify genes encoding the enzymes involved in metabolism of hydroxybenzenes and hydroxybenzoates, we performed BLASTP searches of the C. parapsilosis genome sequence using bacterial protein queries (e.g. NahG, NagIKL, HadC, TbuD and XlnDEF from Ralstonial Pseudomonas species) and the hits were further analysed for the presence of conserved protein domains. These searches revealed two ORFs for homologues of salicylate monooxygenase, i.e. CPAG01781 (MNX1) and CPAG03410 (MNX2). Since C. parapsilosis does not grow on salicylate (see above), we speculated that these ORFs code for 

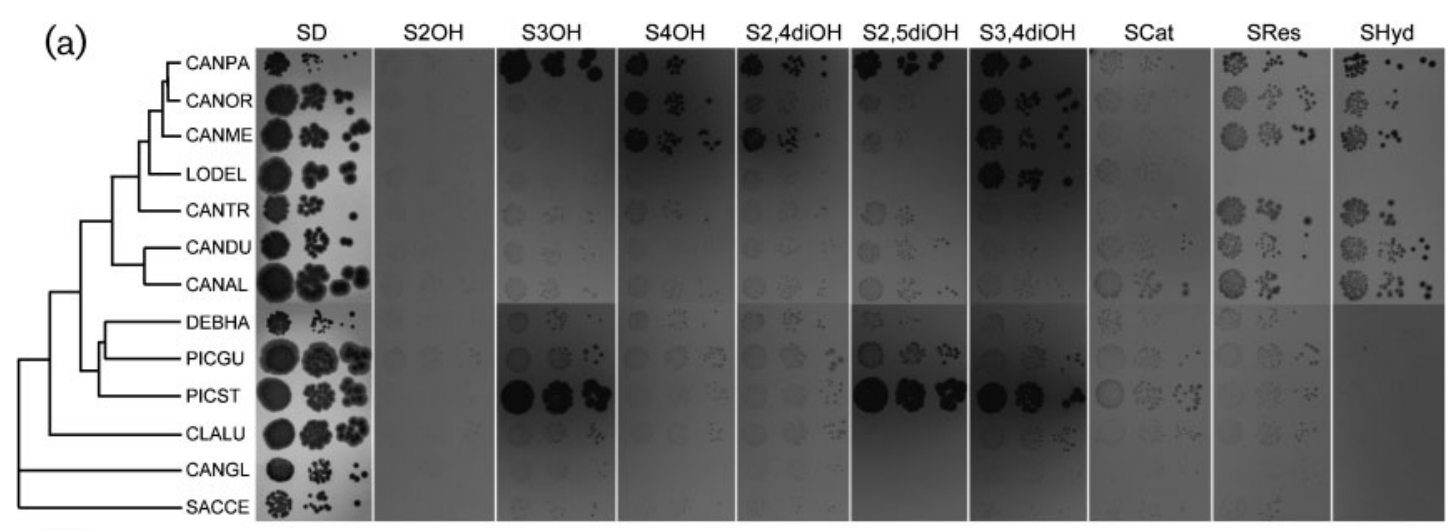

(b)

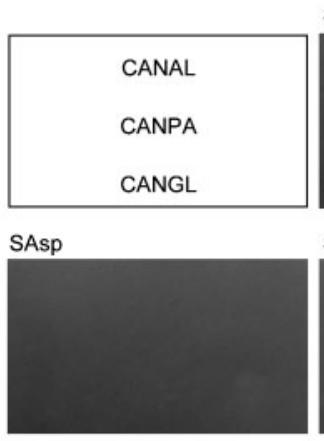

SD

SGly
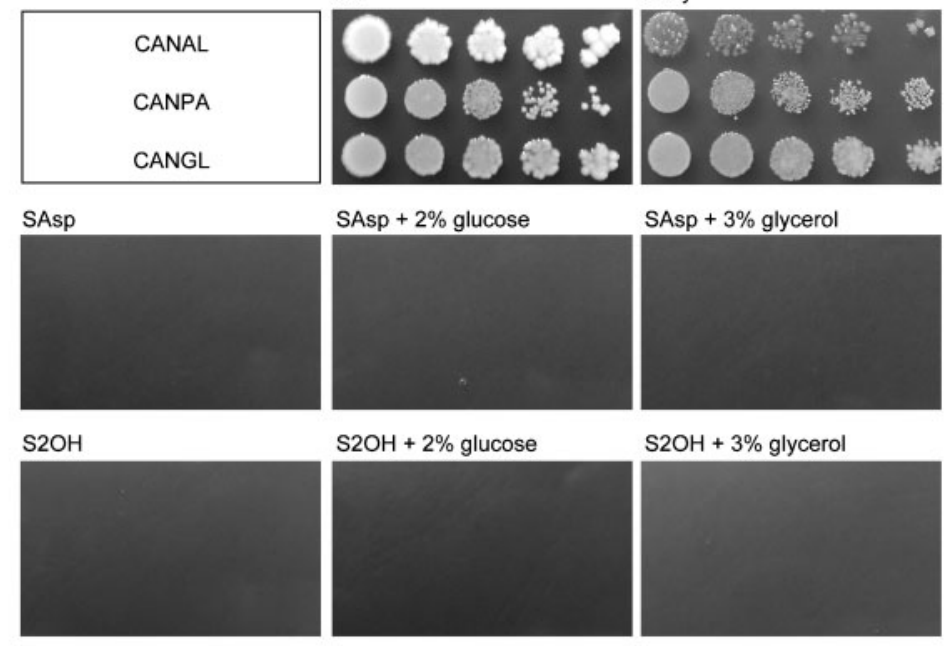

SAsp $+2 \%$ glucose

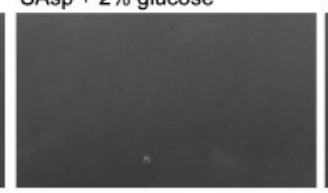

SAsp $+3 \%$ glycerol

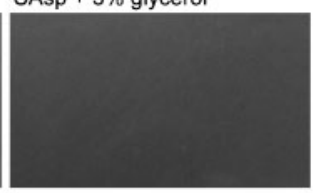

$\mathrm{S} 2 \mathrm{OH}+2 \%$ glucose

$\mathrm{S} 2 \mathrm{OH}+3 \%$ glycerol
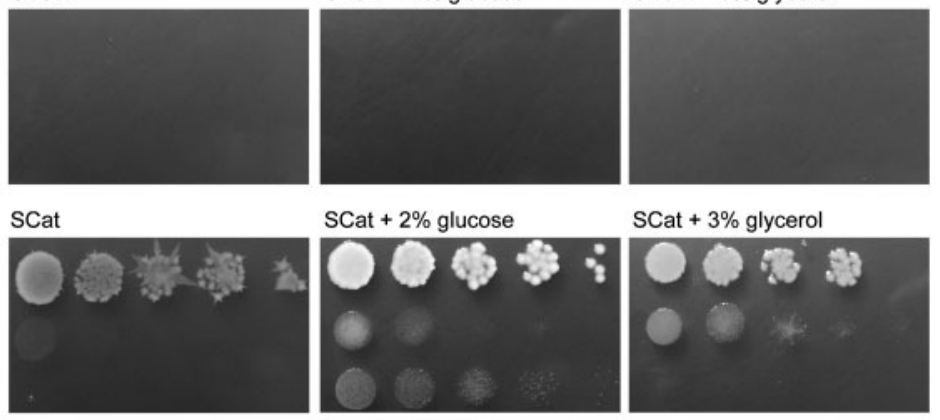

SCat $+2 \%$ glucose

SCat $+3 \%$ glycerol
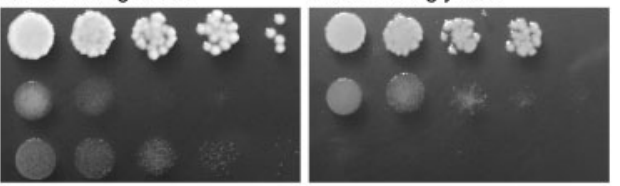

(c)

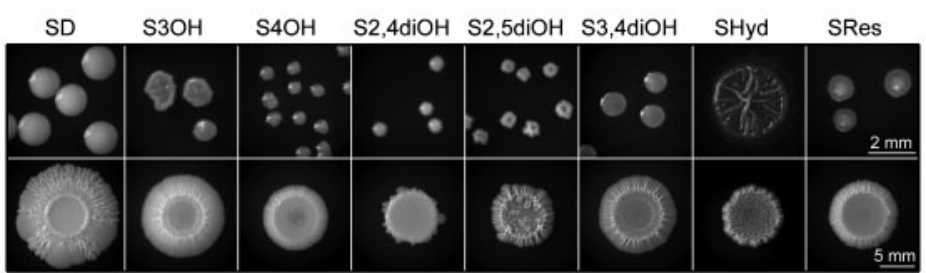

Fig. 2. Utilization of hydroxybenzoates and hydroxybenzenes by C. parapsilosis CLIB214 and selected yeast species. (a) C. parapsilosis (CANPA), C. orthopsilosis (CANOR), C. metapsilosis (CANME), L. elongisporus (LODEL), C. tropicalis (CANTR), C. dubliniensis (CANDU), C. albicans (CANAL), D. hansenii (DEBHA), P. guilliermondii (PICGU), P. stipitis (PICST), Clavispora lusitaniae (CLALU), C. glabrata (CANGL) and S. cerevisiae (SACCE) were grown for 7 days at $28{ }^{\circ} \mathrm{C}$ on the indicated media. Note that media were adjusted using $\mathrm{NaOH}$ to $\mathrm{pH} 6.0-6.5$ and $0.01 \%(\mathrm{w} / \mathrm{v})$ bromothymol blue was used as $\mathrm{pH}$ indicator. Except C. glabrata and S. cerevisiae, the examined species belong to the 'CTG' clade of hemiascomycetes. The phylogenetic relationship among the examined species is indicated. S. cerevisiae strain L5366 has been transformed with pYES2/CT plasmid to complement the ura3-52 mutation and ensure its prototrophy. (b) Aspirin, salicylate and catechol are toxic to C. parapsilosis cells. C. albicans (top row), C. parapsilosis (middle row) and C. glabrata (bottom row) were grown on the indicated media for 3 days at $28{ }^{\circ} \mathrm{C}$. In (a) and (b), the yeast cultures were plated in tenfold serial dilutions. (c) Colony morphology of C. parapsilosis growing from a single cell (clonal colonies) or a suspension of $\sim 10^{5}$ cells (macrocolonies) grown for 21 days at $25{ }^{\circ} \mathrm{C}$ in synthetic media containing the indicated carbon sources. The solid media on plates shown in (b) and (c) did not contain bromothymol blue and their $\mathrm{pH}$ was not adjusted. 


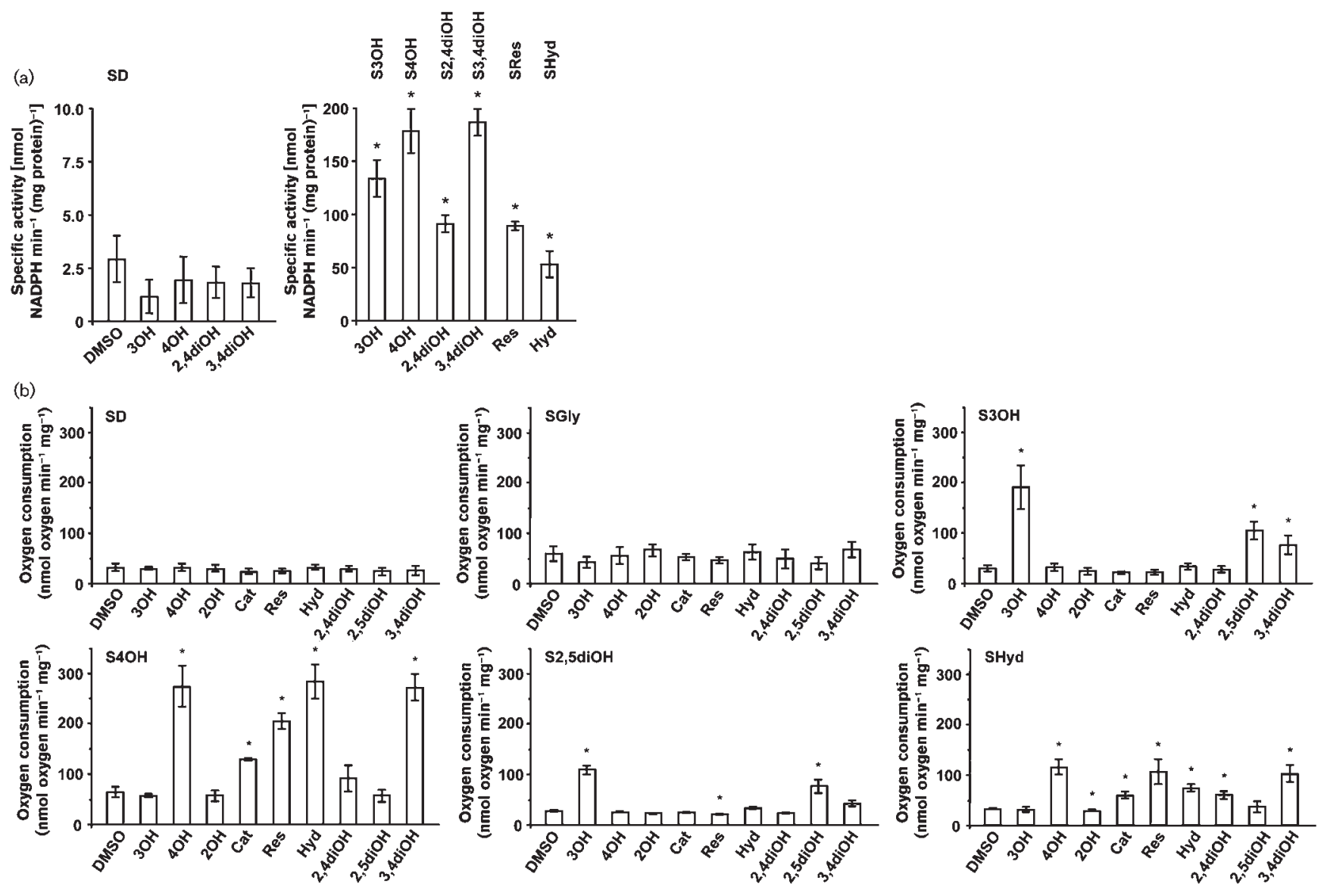

Fig. 3. Enzymatic activities inferred from $N A D(P) H$ oxidation (a) and oxygen consumption (b) assays. C. parapsilosis CLIB214 cells were grown in the indicated synthetic media and processed for analyses of enzymatic activities (see Methods). (a) Flavoprotein monooxygenase activities ascertained by NADPH oxidation in protein extracts were measured after addition of $0.2 \%$ DMSO or $1 \mathrm{mM}$ phenolic substrates, i.e. 3-hydroxybenzoate $(3 \mathrm{OH})$, 4-hydroxybenzoate $(4 \mathrm{OH})$, resorcinate $(2,4 \mathrm{diOH})$, protocatechuate $(3,4 \mathrm{diOH})$, resorcinol (Res) and hydroquinone $(\mathrm{Hyd})$. (b) Oxygen consumption was analysed after addition of $2 \%$ DMSO or $10 \mathrm{mM}$ phenolic substrates, i.e. 3-hydroxybenzoate, 4-hydroxybenzoate, salicylate (2OH), catechol (Cat), resorcinol (Res), hydroquinone, resorcinate, gentisate $(2,5 \mathrm{diOH})$ and protocatechuate. The assays were performed in three independent experiments with two parallel measurements in each case (bars, mean \pm SEM). The significance of differences between the samples and the control (DMSO) was evaluated by Student's $t$-test $\left({ }^{*} P<0.05\right)$.

3-hydroxybenzoate 6-hydroxylase and/or 4-hydroxybenzoate 1-hydroxylase. Although the latter enzyme has been characterized previously (Eppink et al., 1997), both protein products deduced from these ORFs have similar molecular masses of about $50 \mathrm{kDa}[52.6 \mathrm{kDa}(\mathrm{Mnx} 1 \mathrm{p})$ versus $49.9 \mathrm{kDa}$ (Mnx2p)] and the sequence analysis alone did not allow identification of the corresponding enzyme unequivocally. Next, we identified CPAG00562 (MNX3), coding for a homologue of hydroquinone hydroxylase. Our prediction has been confirmed by the presence of peptide sequences determined from the purified protein (Eppink et al., 2000). Moreover, we found candidate ORFs encoding additional components of the investigated pathways such as CPAG03408 (GDX1) and CPAG05217 (HDX1). In the case of fumarylpyruvate hydrolase, we found two candidates, CPAG03407 and CPAG04256. Based on the sequence comparison of deduced protein products we propose that CPAG03407 and CPAG04256 code for fumarylpyruvate hydrolase (FPH1) and fumarylacetoacetate hydrolase (FAA1/ FAH1), respectively (Supplementary Table S1 and Supplementary Fig. S1, available with the online version of this paper). Our search for genes encoding homologues of maleylacetate reductase (MAR) and maleylpyruvate isomerase (MPI) involved in the 3-oxoadipate and gentisate pathway, respectively, revealed multiple candidate ORFs. However, we did not investigate which of them are involved in the analysed pathways.

The ORFs CPAG03410 (MNX2) and CPAG03408 (GDX1) appear to be species-specific, as they do not have counterparts in the C. albicans genome (Logue et al., 2005). Since our results show that $C$. albicans lacks the gentisate 
pathway (Fig. 2a), we assume that MNX2 codes for 3hydroxybenzoate 6-hydroxylase. MNX2 is located within the gene cluster present in the subtelomeric region of the $957321 \mathrm{bp}$ chromosomal contig corresponding to chromosome V (Supplementary Fig. S2). The cluster consists of ORFs coding for presumed components of the gentisate pathway (MNX2, GDX1, FHP1) and three additional ORFs potentially related to gentisate metabolism, i.e. CPAG03409, CPAR873 and CPAG03406, coding for MFS (major facilitator superfamily) transporter, Gfal (glutathione-dependent formaldehyde-activating enzyme) and zinc-finger transcription factor, respectively. Using the Candida gene order browser (Fitzpatrick et al., 2010) we found that the cluster CPAG03410-CPAG03406 is partially conserved in P. stipitis (PICST55211-PICST30014PICST41230-PICST65252), which utilizes substrates metabolized in the gentisate pathway (Fig. 2a). This strengthens the idea that monooxygenase encoded by MNX2 is involved in this pathway. We found that $D$. hansenii also contains two ORFs, DEHA0C01045g and DEHA0C01067g, from this cluster that are orthologues of CPAG03408 (GDX1) and CPAG03407 (FPH1), respectively. However, the deduced protein product of DEHA0C01045g appears to be truncated (Supplementary Fig. S1d), which explains why this yeast does not grow on gentisate (Fig. 2a).

Our search for homologues of MNX1, MNX2, MNX3, GDX1, $H D X 1$ and FPH1 revealed their presence in the genome sequences of yeasts belonging to the 'CTG clade', although most species do not contain the complete set (Supplementary Table S1). This is in line with their inability to utilize corresponding substrates (Fig. 2a). In contrast, the species outside the 'CTG clade' such as C. glabrata, Kluyveromyces lactis, S. cerevisiae, Schizosaccharomyces pombe and Yarrowia lipolytica lack orthologues of all these genes and hence the corresponding enzymatic pathways.

\section{MNX1 and MNX2 code for 4-hydroxybenzoate 1-hydroxylase and 3-hydroxybenzoate 6-hydroxylase, respectively}

Biochemical activities of the protein products encoded by MNX1 and MNX2 were identified as follows. Since we observed that chromosomal copies of the examined genes are not induced in the wild-type cells cultivated in SGal medium (data not shown), we cloned corresponding ORFs into the expression vector pBP7 under the GAL1 promoter. Next, we used the NADPH oxidation assay to detect flavoprotein monooxygenase activities in protein extracts prepared from C. parapsilosis transformants cultivated overnight in SGal medium (Fig. 4). The extracts from cells transformed with the pBP7 vector and the pBP7-MNX3 construct were used as the negative and positive control, respectively. This experiment showed that cells transformed with pBP7-MNX1 exhibied increased enzymatic activities when 4-hydroxybenzoate and protocatechuate were used as substrates in the assay. In contrast, we observed the highest activity with 3-hydroxybenzoate in the extracts prepared from the pBP7-MNX2 transformants. In the case of cells expressing hydroquinone hydroxylase (pBP7-MNX3) we detected increased activities using resorcinol, hydroquinone and catechol. In all three cases, enzymatic activities detected using other substrates were substantially lower or undetectable. Our results indicate that MNX1 and MNX2 code for 4-hydroxybenzoate 1hydroxylase and 3-hydroxybenzoate 6-hydroxylase, respectively. Importantly, the results obtained with MNX1 and MNX3 are consistent with the substrate specificity of the purified enzymes reported by Eppink et al. (1997, 2000).

\section{Hydroxyaromatic compounds induce promoters of MNX1, MNX2, MNX3 and GDX1}

Enzymatic activities involved in the gentisate and 3oxoadipate pathways appear to be regulated independently

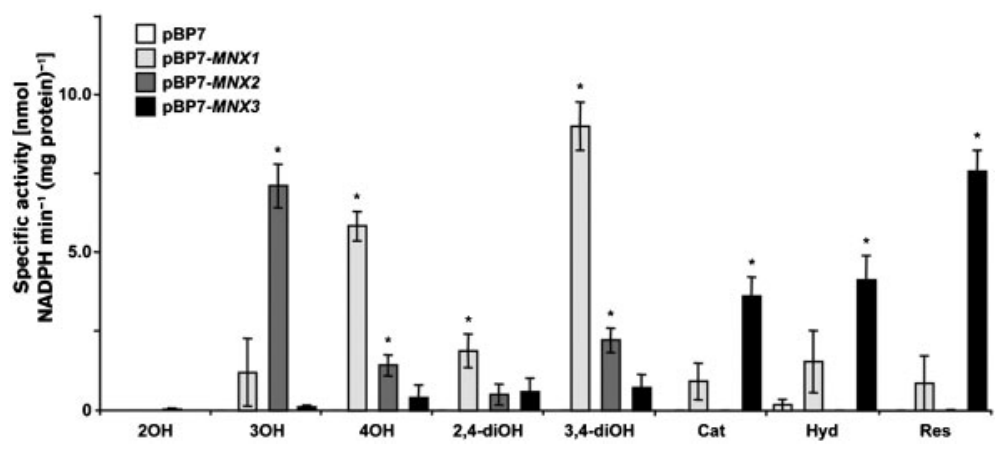

http://mic.sgmjournals.org
Fig. 4. Flavoprotein monooxygenase activities in C. parapsilosis transformants expressing MNX1, MNX2 and MNX3. C. parapsilosis CDU1 cells transformed with pBP7-MNX1, pBP7-MNX2 and pBP7-MNX3 expressing the examined genes under the control of the $G A L 1$ promoter were grown overnight at $28{ }^{\circ} \mathrm{C}$ in SGal medium, then lysed and assayed for NADPH oxidation activity (see Methods) in the presence of $1 \mathrm{mM}$ phenolic substrates, i.e. salicylate $(2 \mathrm{OH}), \quad 3$-hydroxybenzoate $(3 \mathrm{OH}), 4$-hydroxybenzoate $(4 \mathrm{OH})$, resorcinate $(2,4 \mathrm{diOH})$, protocatechuate $(3,4 \mathrm{diOH})$, catechol (Cat), hydroquinone (Hyd) and resorcinol (Res). Transformants with the vector pBP7 were used as the control. The experiment was repeated three times and the data were processed in a similar manner to that described in Fig. 3. 
and are induced by corresponding hydroxyaromatic substrates (Fig. 3). Therefore, we investigated the gene expression using promoter fusions with $\beta$-galactosidase encoded by $K$. lactis gene LAC4. We constructed plasmids derived from the pPK3 vector containing yeast $\beta$ galactosidase driven by promoters of the examined genes. Subsequently, we analysed $\beta$-galactosidase activity in $C$. parapsilosis SR23 met-1 transformants cultivated in synthetic media containing various substrates as the sole carbon sources. Our results show that both pathways are controlled at the level of transcription. The levels of $\beta$ galactosidase in cells cultivated in media with glucose and galactose are relatively low. However, we observed increased activities on hydroxyaromatic substrates (Table 4). For example, we detected very strong induction of $\beta$ galactosidase in transformants containing pPK3- $\mathrm{P}_{M N X 1}$ or pPK3-P $\mathrm{P}_{M N X 3}$. The activity expressed from the MNX1 promoter was $\sim 2300$ times higher in cells grown on 4hydroxybenzoate than on glucose. Importantly, the promoters of gene pairs MNX1-MNX3 and MNX2-GDX1 appear to be co-regulated depending on the substrate. While the former pair of promoters was highly induced on 4-hydroxybenzoate, protocatechuate and resorcinol, the induction of the latter pair was observed on 3hydroxybenzoate and gentisate. This is in line with our conclusion that MNX1 and MNX2 are involved in the 3oxoadipate and gentisate pathways, respectively.

\section{Enzymes of the gentisate and 3-oxoadipate pathways differ by intracellular localization}

We expressed recombinant proteins Mnx1p, Mnx2p, Mnx3p and Gdx1p tagged at their C-termini with yEGFP3 to identify their intracellular localization. First, we prepared a set of plasmid constructs derived from the pBP8 vector. These constructs contain corresponding ORFs lacking the terminal codon fused in-frame with the sequence coding for yEGFP3. Next, we transformed the plasmids into C. parapsilosis CDU1 cells and cultivated the transformants in synthetic media. Using fluorescence microscopy we observed the fusion proteins Mnx1-yEGFP3 and Mnx3-yEGFP3 in the cytosol. In contrast, we found that localization of Mnx2-yEGFP3 and Gdx1-yEGFP3 depends on the corresponding substrate present in the medium. In SD medium, we detected both proteins in the cytosol. However, in media containing 3hydroxybenzoate or gentisate as the sole carbon source, we detected the fusion proteins as discrete spots or foci. Subsequent staining with MitoTracker Red revealed that they co-localize with mitochondria (Fig. 5). This indicates that enzymes of the gentisate pathway are associated with mitochondria in a substrate-dependent manner, although the molecular mechanism controlling intracellular distribution of these proteins remains unknown. Nevertheless, we assume that the gentisate pathway components associate with mitochondria in the wild-type cells, as the expression of corresponding enzymes is induced only in media with hydroxyaromatic substrates. Since Mnx2p and Gdx1p do not have a recognizable mitochondrial import sequence, the
Table 4. Promoter activities of $M N X 1, M N X 2, M N X 3$ and $G D X 1$ genes were assayed in whole-cell lysates of $C$. parapsilosis SR23 met-1 transformed with corresponding plasmid constructs

The cultures were grown to $\mathrm{OD}_{600} 1$ at $28{ }^{\circ} \mathrm{C}$ in the indicated medium supplemented with adenine and lysine. Transformants containing the pPK3 vector lacking a promoter were used as a negative control. $\beta$ Galactosidase activity was determined essentially as described by Rose \& Botstein (1983). Each value represents the mean \pm SEM from three independent experiments. Note that strain SR23 met-1 does not grow in SHyd medium so we did not test the effect of hydroquinone on the genes expression.

\begin{tabular}{|c|c|c|}
\hline $\begin{array}{l}\text { Growth } \\
\text { medium }\end{array}$ & $\begin{array}{l}\text { Plasmid } \\
\text { construct }\end{array}$ & $\begin{array}{l}\text { Specific } \beta \text {-galactosidase } \\
\text { activity (Miller units) }\end{array}$ \\
\hline \multirow[t]{4}{*}{$\mathrm{SD}$} & pPK3-P ${ }_{M N X 1}$ & $1.45 \pm 0.15$ \\
\hline & $\mathrm{pPK} 3-\mathrm{P}_{M N X 2}$ & $1.66 \pm 0.04$ \\
\hline & $\mathrm{pPK} 3-\mathrm{P}_{M N X 3}$ & $13.20 \pm 0.49$ \\
\hline & $\mathrm{pPK} 3-\mathrm{P}_{G D X 1}$ & $1.47 \pm 0.28$ \\
\hline \multirow[t]{4}{*}{ SGal } & $\mathrm{pPK} 3-\mathrm{P}_{M N X 1}$ & $0.75 \pm 0.36$ \\
\hline & $\mathrm{pPK} 3-\mathrm{P}_{M N X 2}$ & $1.09 \pm 0.01$ \\
\hline & pPK3-P ${ }_{M N X 3}$ & $17.24 \pm 1.10$ \\
\hline & $\mathrm{pPK} 3-\mathrm{P}_{G D X 1}$ & $1.76 \pm 0.53$ \\
\hline \multirow[t]{4}{*}{$\mathrm{S} 3 \mathrm{OH}$} & pPK3-P ${ }_{M N X 1}$ & $3.33 \pm 0.50$ \\
\hline & $\mathrm{pPK} 3-\mathrm{P}_{M N X 2}$ & $14.78 \pm 0.32$ \\
\hline & $\mathrm{pPK} 3-\mathrm{P}_{M N X 3}$ & $17.32 \pm 0.22$ \\
\hline & $\mathrm{pPK} 3-\mathrm{P}_{G D X 1}$ & $423.13 \pm 37.96$ \\
\hline \multirow[t]{4}{*}{$\mathrm{S} 4 \mathrm{OH}$} & $\mathrm{pPK} 3-\mathrm{P}_{M N X 1}$ & $3412.38 \pm 129.81$ \\
\hline & pPK3-P $\mathrm{P}_{M N X 2}$ & $3.87 \pm 0.42$ \\
\hline & $\mathrm{pPK} 3-\mathrm{P}_{M N X 3}$ & $5154.72 \pm 196.34$ \\
\hline & pPK3-P ${ }_{G D X 1}$ & $13.23 \pm 0.82$ \\
\hline \multirow[t]{4}{*}{$\mathrm{S} 2,5 \mathrm{diOH}$} & $\mathrm{pPK} 3-\mathrm{P}_{M N X 1}$ & $3.89 \pm 0.37$ \\
\hline & $\mathrm{pPK} 3-\mathrm{P}_{M N X 2}$ & $5.11 \pm 0.54$ \\
\hline & $\mathrm{pPK} 3-\mathrm{P}_{M N X 3}$ & $13.19 \pm 0.37$ \\
\hline & $\mathrm{pPK} 3-\mathrm{P}_{G D X 1}$ & $698.81 \pm 77.99$ \\
\hline \multirow[t]{4}{*}{$\mathrm{S} 3,4 \mathrm{diOH}$} & $\mathrm{pPK} 3-\mathrm{P}_{M N X 1}$ & $1943.89 \pm 122.88$ \\
\hline & pPK3-P ${ }_{M N X 2}$ & $0.62 \pm 0.27$ \\
\hline & $\mathrm{pPK} 3-\mathrm{P}_{M N X 3}$ & $2146.17 \pm 200.03$ \\
\hline & $\mathrm{pPK} 3-\mathrm{P}_{G D X 1}$ & $7.11 \pm 0.78$ \\
\hline \multirow[t]{4}{*}{ SRes } & $\mathrm{pPK} 3-\mathrm{P}_{M N X 1}$ & $998.37 \pm 119.07$ \\
\hline & $\mathrm{pPK} 3-\mathrm{P}_{M N X 2}$ & $1.12 \pm 0.06$ \\
\hline & $\mathrm{pPK} 3-\mathrm{P}_{M N X 3}$ & $3083.31 \pm 436.53$ \\
\hline & $\mathrm{pPK} 3-\mathrm{P}_{G D X 1}$ & $5.61 \pm 0.25$ \\
\hline
\end{tabular}

question remains whether they are imported into mitochondria or attached to the mitochondrial outer membrane. The mitochondrial localization of the gentisate pathway is also supported by the putative $\mathrm{N}$-terminal mitochondrial import signal found in Fphlp, which acts downstream of Gdxlp in the pathway. Taken together, we conclude that the 3oxoadipate pathway operates in the cytosol and the gentisate pathway in, or in close proximity to, the mitochondria.

\section{MNX1 and MNX2 increase the tolerance of C. parapsilosis to terbinafine}

C. parapsilosis isolates are highly sensitive to the antifungal drug terbinafine (lamisil), an inhibitor of squalene 
$\mathrm{SD}$

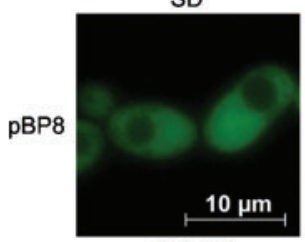

yEGFP3

SD

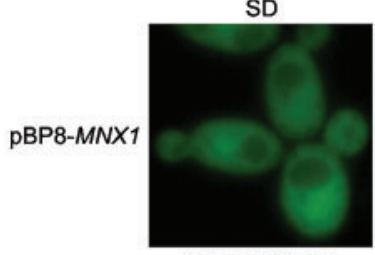

Mnx1-yEGFP3

SD

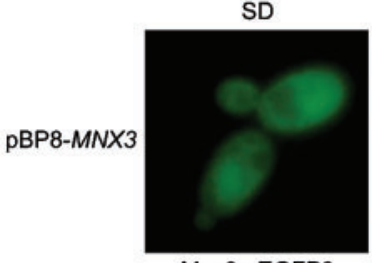

Mnx3-yEGFP3
$\mathrm{S} 4 \mathrm{OH}$

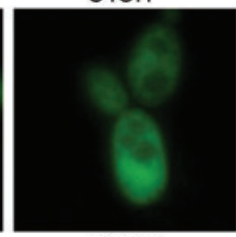

yEGFP3

$\mathrm{S} 4 \mathrm{OH}$

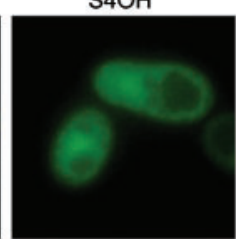

Mnx1-yEGFP3

SHyd

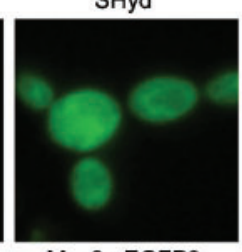

Mnx3-yEGFP3

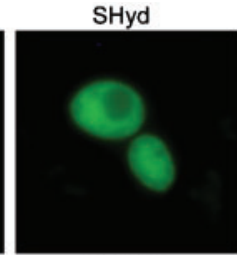

yEGFP3
$\mathrm{S} 3 \mathrm{OH}$

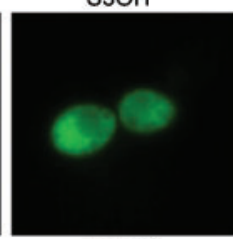

yEGFP3
S2,5diOH

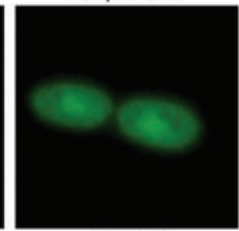

yEGFP3

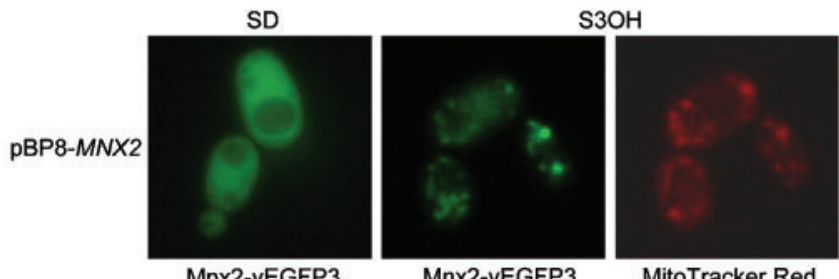

Mnx2-yEGFP3

SD

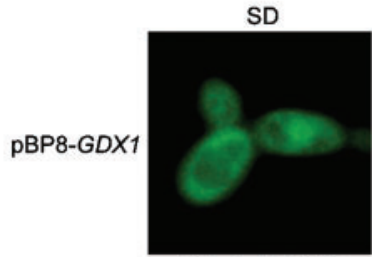

Gdx1-yEGFP3

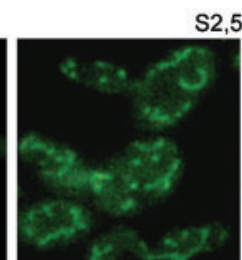

Gdx1-yEGFP3
S2,5diOH

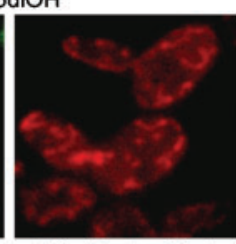

Fig. 5. Intracellular localization of Mnx1p, Mnx2p, Mnx3p and Gdx1p. C. parapsilosis CDU1 transformed with pBP8-MNX1, pBP8-MNX2, pBP8-MNX3 and pBP8-GDX1 expressing the examined proteins tagged with yEGFP3 were grown at $28{ }^{\circ} \mathrm{C}$ for $48 \mathrm{~h}$ in synthetic media containing the indicated carbon sources and examined by fluorescence microscopy (Olympus BX50). Transformants with the vector pBP8 were used as a control. Mitochondria were stained with MitoTracker Red CMXRos.

epoxidase involved in ergosterol biosynthesis (Ryder et al., 1998). Understanding the possible causes of the drug resistance is therefore important for clinical microbiology. It has been reported that salicylate monooxygenase encoded by the salA gene mediates the resistance of Aspergillus nidulans to terbinafine (Graminha et al., 2004). These authors proposed that the naphthalene nucleus of terbinafine is degraded to salicylate or a similar product. In bacteria, naphthalene can be metabolized via salicylate and gentisate (Grund et al., 1992). Therefore, we examined whether MNX1 and MNX2 contribute to the survival of $C$. parapsilosis treated with terbinafine. We cultivated $C$. parapsilosis CDU1 cells transformed with pBP7, pBP7MNX1 and pBP7-MNX2 in SGal media containing increasing amounts of terbinafine for $48 \mathrm{~h}$ and observed that cells expressing MNX1 and MNX2 under the GAL1 promoter exhibit increased tolerance to terbinafine (Fig. 6). This suggests that degradation of terbinafine in $C$. parapsilosis proceeds via both 3-oxoadipate and gentisate pathways. We did not observe Mnx1p- and/or Mnx2p-

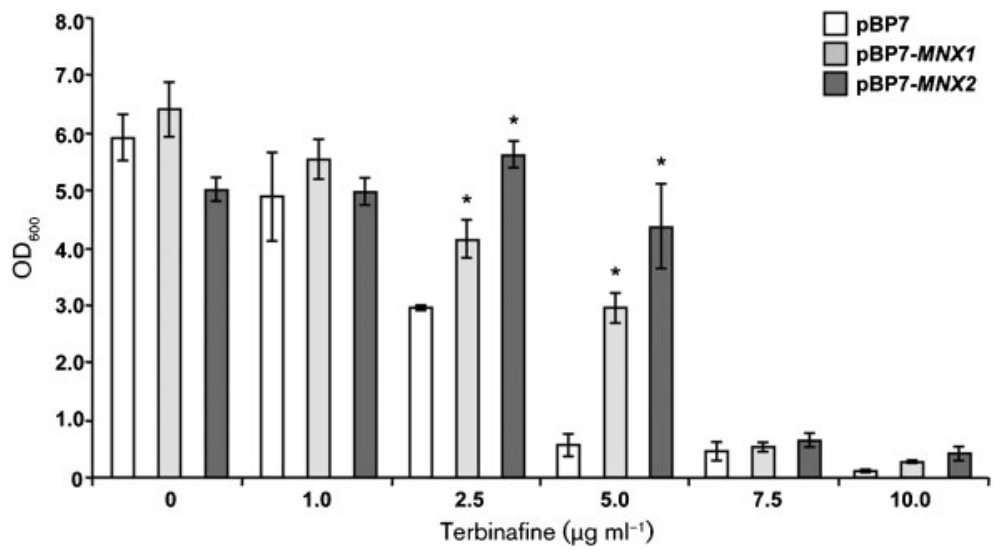

Fig. 6. $M N X 1$ and $M N X 2$ increase tolerance of C. parapsilosis cells to terbinafine. C. parapsilosis CDU1 transformed with pBP7, pBP7$M N X 1$ and pBP7-MNX2 were grown in liquid SGal medium containing terbinafine $(0,1.0$, 2.5, 5.0, 7.5 and $10 \mu \mathrm{g} \mathrm{ml}^{-1}$ ) for $48 \mathrm{~h}$ at $28{ }^{\circ} \mathrm{C}$. The growth yield was determined by measurement of $\mathrm{OD}_{600}$. The experiment was performed twice (bars, mean \pm SEM). The statistical analysis was performed using the twofactor analysis of variance (ANOVA) test $\left({ }^{*} P<0.05\right)$. 
mediated degradation of salicylate (Fig. 4). This corresponds to the inability of $C$. parapsilosis to use salicylate and catechol as the growth substrates. However, we demonstrate that catechol is utilized as a substrate in the assays shown in Figs 3(b) and 4, presumably due to the activity of hydroquinone hydroxylase, which has low affinity $\left(K_{\mathrm{m}}=500 \mu \mathrm{M}\right)$ for this substrate (Eppink et al., 2000). Although in the presence of glucose C. parapsilosis wild-type cells do not express MNX1 and MNX2 and are susceptible to terbinafine, our experiment demonstrates that the activation of these genes may generate cells tolerant to higher doses of this drug.

\section{Conclusions}

C. parapsilosis metabolizes hydroxybenzenes and hydroxybenzoates using inducible gentisate and 3-oxoadipate pathways. We propose that catabolic degradation of these compounds may have a particular role in the physiology of C. parapsilosis and could provide a growth advantage in a specific ecological niche. Both pathways can contribute to the survival of yeast cells in an environment enriched in phenolic derivatives (e.g. lignin degradation products) and can potentially mediate the pathogen's tolerance to antifungal treatment with terbinafine. In contrast with $C$. parapsilosis, the range of hydroxyaromatic compounds utilized as the sole carbon sources differs in closely related species. Comparative analysis of the complete genome sequences revealed that most yeast species lack homologues coding for enzymes involved in these pathways. This evokes questions concerning evolutionary and ecological constraints leading to diversity in the catabolism of phenolic compounds observed in the 'CTG' clade species. Further studies should address how these pathways and corresponding genes evolved in hemiascomycetes and whether they were present in their common ancestor and subsequently lost in most lineages or, alternatively, entered the 'CTG' clade relatively recently by horizontal gene transfer.

\section{ACKNOWLEDGEMENTS}

We would like to thank Ladislav Kovac for continuous support, colleagues from our laboratory for discussions and critical reading of the manuscript, and G. Butler and C. Ding (Conway Institute, Dublin, Ireland) for providing us with C. parapsilosis strains CLIB214 and CDU1. This work was supported by grants from the Howard Hughes Medical Institute (55005622), the Slovak grant agencies VEGA (1/0219/08, 1/0132/09) and APVV (0024-07) and the Comenius University (UK/276/2009).

\section{REFERENCES}

Balzan, R., Sapienza, K., Galea, D. R., Vassallo, N., Frey, H. \& Bannister, W. H. (2004). Aspirin commits yeast cells to apoptosis depending on carbon source. Microbiology 150, 109-115.

Butler, G., Rasmussen, M. D., Lin, M. F., Santos, M. A., Sakthikumar, S., Munro, C. A., Rheinbay, E., Grabherr, M., Forche, A. \& other authors
(2009). Evolution of pathogenicity and sexual reproduction in eight Candida genomes. Nature 459, 657-662.

Carruba, G., Pontieri, E., De Bernardis, F., Martino, P. \& Cassone, A. (1991). DNA fingerprinting and electrophoretic karyotype of environmental and clinical isolates of Candida parapsilosis. J Clin Microbiol 29, 916-922.

Cooper, B. H. \& Land, G. A. (1979). Assimilation of protocatechuic acid and $p$-hydroxybenzoic acid as an aid to laboratory identification of Candida parapsilosis and other medically important yeasts. J Clin Microbiol 10, 343-345.

Ding, C. \& Butler, G. (2007). Development of a gene knockout system in Candida parapsilosis reveals a conserved role for BCR1 in biofilm formation. Eukaryot Cell 6, 1310-1319.

Eppink, M. H., Boeren, S. A., Vervoort, J. \& van Berkel, W. J. (1997). Purification and properties of 4-hydroxybenzoate 1-hydroxylase (decarboxylating), a novel flavin adenine dinucleotide-dependent monooxygenase from Candida parapsilosis CBS604. J Bacteriol 179, 6680-6687.

Eppink, M. H., Cammaart, E., Van Wassenaar, D., Middelhoven, W. J. \& van Berkel, W. J. (2000). Purification and properties of hydroquinone hydroxylase, a FAD-dependent monooxygenase involved in the catabolism of 4-hydroxybenzoate in Candida parapsilosis CBS604. Eur J Biochem 267, 6832-6840.

Fitzpatrick, D. A., O'Gaora, P., Byrne, K. P. \& Butler, G. (2010). Analysis of gene evolution and metabolic pathways using the Candida Gene Order Browser. BMC Genomics 11, 290.

Gimeno, C. J. \& Fink, G. R. (1994). Induction of pseudohyphal growth by overexpression of PHD1, a Saccharomyces cerevisiae gene related to transcriptional regulators of fungal development. Mol Cell Biol 14, 2100-2112.

Graminha, M. A., Rocha, E. M., Prade, R. A. \& Martinez-Rossi, N. M. (2004). Terbinafine resistance mediated by salicylate 1-monooxygenase in Aspergillus nidulans. Antimicrob Agents Chemother 48, 35303535.

Grund, E., Denecke, B. \& Eichenlaub, R. (1992). Naphthalene degradation via salicylate and gentisate by Rhodococcus sp. strain B4. Appl Environ Microbiol 58, 1874-1877.

Jeffries, T. W., Grigoriev, I. V., Grimwood, J., Laplaza, J. M., Aerts, A., Salamov, A., Schmutz, J., Lindquist, E., Dehal, P. \& other authors (2007). Genome sequence of the lignocellulose-bioconverting and xylose-fermenting yeast Pichia stipitis. Nat Biotechnol 25, 319-326.

Jones, T., Federspiel, N. A., Chibana, H., Dungan, J., Kalman, S., Magee, B. B., Newport, G., Thorstenson, Y. R., Agabian, N. \& other authors (2004). The diploid genome sequence of Candida albicans. Proc Natl Acad Sci U S A 101, 7329-7334.

Kosa, P., Gavenciakova, B. \& Nosek, J. (2007). Development of a set of plasmid vectors for genetic manipulations of the pathogenic yeast Candida parapsilosis. Gene 396, 338-345.

Leeuw, N. J., Swart, C. W., Ncango, D. M., Pohl, C. H., Sebolai, O. M., Strauss, C. J., Botes, P. J., van Wyk, P. W., Nigam, S. \& Kock, J. L. (2007). Acetylsalicylic acid as antifungal in Eremothecium and other yeasts. Antonie van Leeuwenhoek 91, 393-405.

Logue, M. E., Wong, S., Wolfe, K. H. \& Butler, G. (2005). A genome sequence survey shows that the pathogenic yeast Candida parapsilosis has a defective MTLal allele at its mating type locus. Eukaryot Cell 4, 1009-1017.

Middelhoven, W. J. (1993). Catabolism of benzene compounds by ascomycetous and basidiomycetous yeasts and yeastlike fungi. A literature review and an experimental approach. Antonie van Leeuwenhoek 63, 125-144.

Middelhoven, W. J., Coenen, A., Kraakman, B. \& Sollewijn Gelpke, M. D. (1992). Degradation of some phenols and hydroxybenzoates by 
the imperfect ascomycetous yeasts Candida parapsilosis and Arxula adeninivorans: evidence for an operative gentisate pathway. Antonie van Leeuwenhoek 62, 181-187.

Nosek, J., Adamikova, L., Zemanova, J., Tomaska, L., Zufferey, R. \& Mamoun, C. B. (2002). Genetic manipulation of the pathogenic yeast Candida parapsilosis. Curr Genet 42, 27-35.

Nosek, J., Holesova, Z., Kosa, P., Gacser, A. \& Tomaska, L. (2009). Biology and genetics of the pathogenic yeast Candida parapsilosis. Curr Genet 55, 497-509.

Rose, M. \& Botstein, D. (1983). Construction and use of gene fusions to lacZ (beta-galactosidase) that are expressed in yeast. Methods Enzymol 101, 167-180.

Ryder, N. S., Wagner, S. \& Leitner, I. (1998). In vitro activities of terbinafine against cutaneous isolates of Candida albicans and other pathogenic yeasts. Antimicrob Agents Chemother 42, 1057-1061.

Suh, S. O., Nguyen, N. H. \& Blackwell, M. (2008). Yeasts isolated from plant-associated beetles and other insects: seven novel Candida species near Candida albicans. FEMS Yeast Res 8, 88-102.

Tavanti, A., Davidson, A. D., Gow, N. A., Maiden, M. C. \& Odds, F. C. (2005). Candida orthopsilosis and Candida metapsilosis spp. nov. to replace Candida parapsilosis groups II and III. J Clin Microbiol 43, 284-292.
Trindade, R. C., Resende, M. A., Silva, C. M. \& Rosa, C. A. (2002). Yeasts associated with fresh and frozen pulps of Brazilian tropical fruits. Syst Appl Microbiol 25, 294-300.

Trofa, D., Gácser, A. \& Nosanchuk, J. D. (2008). Candida parapsilosis, an emerging fungal pathogen. Clin Microbiol Rev 21, 606-625.

van Asbeck, E. C., Clemons, K. V. \& Stevens, D. A. (2009). Candida parapsilosis: a review of its epidemiology, pathogenesis, clinical aspects, typing and antimicrobial susceptibility. Crit Rev Microbiol 35, 283-309.

van Berkel, W. J., Eppink, M. H., Middelhoven, W. J., Vervoort, J. \& Rietjens, I. M. (1994). Catabolism of 4-hydroxybenzoate in Candida parapsilosis proceeds through initial oxidative decarboxylation by a FAD-dependent 4-hydroxybenzoate 1-hydroxylase. FEMS Microbiol Lett 121, 207-215.

Weems, J. J., Jr (1992). Candida parapsilosis: epidemiology, pathogenicity, clinical manifestations, and antimicrobial susceptibility. Clin Infect Dis 14, 756-766.

Zemanova, J., Nosek, J. \& Tomaska, L. (2004). High-efficiency transformation of the pathogenic yeast Candida parapsilosis. Curr Genet 45, 183-186.

Edited by: K. Kuchler 